\title{
De la metadona al LAAM: Nuevos perfiles terapéuticos. Utilidad de la monitorización terapéutica (MT)
}

\author{
Baño, Ma D.*; López, Ma L.*; Agujetas, M.*; Muñoz „V.*; Guillén, J. L.** \\ * Programa Municipal de Drogas. Majadahonda .Madrid. \\ * Facultad de Farmacia. Escuela de análisis clínicos.(UCM).

\section{RESUMEN:}

La introducción del LAAM en el tratamiento farmacológico de la dependencia a opiáceos, supone un avance importante, al poder diversificar el uso de opioides que hasta el momento, en nuestro país, se había reducido a la metadona.

El objetivo de este trabajo ha sido la identificación de nuevos perfiles de uso del LAAM, teniendo en cuenta aspectos farmacocinéticos y posibles interacciones con otros fármacos y apoyado por los resultados obtenidos sobre niveles plasmáticos de metadona y su posible aplicación al LAAM.

En el momento actual, su perfil de uso sólo ha sido para pacientes en metadona estabilizados, con buena respuesta al tratamiento, y a los que se deseaba flexibilizar y espaciar la administración diaria . En este artículo se proponen otros perfiles de uso, tales como programas de alto umbral orientados al cambio con desintoxicación y posterior paso a programa libre de drogas y sobre todo pacientes en metadona que pierden estabilidad y presentan síndrome de abstinencia a opiáceos, con disminución de los niveles plasmáticos de metadona, debido a interacciones medicamentosas o a otros motivos.

Será necesario realizar estudios con los perfiles propuestos, que amplíen el uso del LAAM para pacientes que tienen problemas con la metadona, como una alternativa terapeútica, complementada con la monitorización en plasma como un instrumento de ayuda y evaluación que mejore la eficacia de los mismos.

Palabras Clave: metadona, LAAM, niveles plasmáticos de metadona, farmacocinética, nuevos perfiles.

\section{ABSTRACT:}

The introduction of LAAM in the treatment of opiates dependence is an important advance that allows to diversify the use of opiates substitutes because, by the moment, methadone had been the only therapy of this type in our country.

The objective of this work has been the identification of new profiles of patients for the use of LAAM, taking into account pharmacokinetic issues and possible interactions with other drugs, using the information given by methadone levels in plasma and its possible application to LAAM.

At present, the profile for the use of LAAM is limited for stable patients with methadone, with good response to the treatment, in order to make more flexible and to space out the daily dosing. In this article, we propose other profiles of use, as high border programs pointed towards the change to the curing of drug addiction and then going into a free-drug program, and in patients who lose the stability and show opiates withdrawal syndrome, with a decrease of methadone levels in plasma due to drug-drug interactions or other reasons.

Other studies with the proposed profiles will be necessary, in order to extend the treatment with LAAM for patients who have clinical problems with methadone, as an alternative treatment, using therapeutic drug monitoring in plasma as an assistance and evaluation tool to improve the effectiveness of these therapies.

Key words: methadone, LAAM, methadone plasma levels, pharmacokinetic, new profiles of patients.

\section{INTRODUCCIÓN}

$\mathbf{L}$ os Programas de Mantenimiento con Metadona (PMM) han sido los más rigurosamente estudiados en los últimos años y los que han aportado resultados más efectivos en el tratamiento y segui- miento de pacientes adictos a opiáceos (Wolff y Strang 1999).

Diferentes estudios (Hall et al, 1993, Ward et al, 1992) reflejan beneficios adicionales para los PMM que incluyen una estabilización de la dependencia de los pacientes, acompañado de una mejoría en las relaciones familiares, (Zannini et al,1997), reducción en el 
uso de drogas intravenosas (Schuster, 1991), mejoras laborales (Ball et al, 1988), en su estado general de salud y calidad de vida (Reno y Aiken, 1993), (Zannini et al, 1997) reducción del riesgo de infección por VIH, hepatitis B y C a través de la vía inyectada (Kang et al, 1993), (Mc Lachlan et al, 1993), (Stimmel et al, 1993) y mejor coste-eficacia comparado con otras alternativas terapeúticas (Glass, 1993),aunque está demostrado que la efectividad de los programas de metadona está mas relacionada con la organización de los mismos que con las características de los pacientes (Brugal y Puigdollers 1999).

Las revisiones americanas tienden, en general, a considerar a la metadona como un tratamiento farmacológico directo, de la misma manera que la insulina se usa para la diabetes (Seivewright y Greenwood, 1996)

La autorización del LAAM en 1993 por la FDA para el tratamiento de la dependencia de opiáceos (Rawson y Ling,1993), (Nightingale, 1993) supone un claro avance en la oferta terapeútica de estos trastornos, y significa el inicio de la normalización de la farmacopea actual en los programas de mantenimiento con opiáceos al poder diversificar los medicamentos utilizables que, hasta el momento, en nuestro país, se habían reducido exclusivamente a la metadona. En el momento actual el uso del LAAM está autorizado en todos los países de la CEE.

El LAAM es sólo el primero de lo que será una serie de ofertas terapeúticas en los programas con sustitutivos opiáceos, pudiendo ser utilizado como una alternativa a la metadona. Los centros asistenciales deberán tener capacidad para utilizar estrategias farmacológicas diversas, para atender las diferentes necesidades de la población adicta.

Por otra parte, los avances técnicos realizados en los últimos años, están sentando los principios de la monitorización terapeútica (MT) de los fármacos, comenzando con la medición de niveles de metadona para pacientes en dichos programas como un instrumento de ayuda en la estabilización del paciente. Es necesario que los clínicos tengan acceso a este tipo de determinaciones que les permitan conocer los niveles plasmáticos de metadona en cada paciente y sus cambios en el tiempo (Wolff, 1991), (Baño et al, 2000).

Mientras esto ocurre, los clínicos están trabajando sin esta herramienta, de la misma manera que los diabetólogos en circunstancias extremas pueden trabajar sin controles de glucosa.

En este artículo revisaremos las indicaciones terapeúticas de LAAM, identificación de nuevos perfiles, teniendo en cuenta los aspectos farmacocinéticos y posibles interacciones con otros fármacos, los resultados obtenidos de la MT hasta el momento actual en relación a la metadona y su posible aplicación con el LAAM.

\section{FARMACOLOGÍA CLÍNICA DE METADONA Y LAAM}

La administración de metadona y LAAM inicia una serie de procesos independientes que determinan en cada momento la concentración de los mismos en el lugar donde ejercen su acción; estos procesos son absorción, distribución, metabolismo y excreción.

\section{METADONA}

La metadona es un agonista opioide tipo "mu", que fue sintetizado en Alemania en los años 40 y se usa desde hace tres décadas como tratamiento de sustitución en la dependencia y el síndrome de abstinencia inducidos por la heroína.

Los estudios publicados demuestran que el tiempo de aparición de metadona en plasma es de 10 minutos después de su inyección por vía subcutánea. Debido a sus características de base débil ( $\mathrm{pKa}$ ) y liposolubilidad, presenta una buena absorción gastrointestinal y después de su administración oral el tiempo de aparición en plasma es de 30 minutos, alcanzándose las concentraciones máximas al cabo de 4 horas. Cambios en el pH gástrico pueden producir modificaciones en los parámetros farmacocinéticos y farmacodinámicos (De Castro, 1996).

Debido a la gran liposolubilidad que presenta la metadona, se distribuye ampliamente por todo el organismo, en especial en cerebro, hígado, pulmón, bazo y riñón, lo que hace que tras la administración repetida se alcancen concentraciones estables en plasma, prolongando su semivida. A este hecho también contribuye su alto grado de unión a proteínas plasmáticas (unión del $90 \%$ a la alfa1 glicoproteína ácida) que actúa como reservorio del fármaco, limitando su concentración libre y prolongando sus acciones farmacológicas en los pacientes que reciben una dosis diaria de mantenimiento (Jaffe, 1990).

Factores que produzcan una disminución en la distribución de la metadona, implicarían un aumento en los niveles del fármaco, con el consiguiente aumento en sus efectos de refuerzo y depresores centrales y viceversa.

Las concentraciones encefálicas máximas de metadona aparecen una o dos horas después de su administración por vía subcutánea o intramuscular, lo que se correlaciona con la intensidad y duración de sus efectos analgésicos (Jaffe, 1990). 
La vida media plasmática de la metadona tras la administración crónica pasa a ser de 15-47 h, con una media de 25 h (McEvoy, 1990). Al suspender su administración, su presencia continúa durante algún tiempo en la sangre, debido a su liberación lenta desde los tejidos que actúan como reservorio del fármaco, lo que explica que el síndrome de abstinencia sea moderado ( Baño et al, 1998).

Se metaboliza en hígado a través de enzimas microsomales hepáticas, produciéndose, por N-demetilación, seguida de ciclación, metabolitos más polares farmacológicamente inactivos, que se excretan a través de la bilis y la orina. El metabolito principal es $\mathrm{N}$ demetilmetadona, que se cicla rápidamente, se deshidrata e isomeriza a una pirrolidina inactiva "EDDP" (2-etileno-1,5 dimetil 3-3 difenilpirrolodina). El metabolito secundario formado por una di-N-demetilación,es "EMDP" (2 etil 5 metil 3-3 difenil pirrolina).

La metadona además, es capaz de acelerar su propio metabolismo (Wolff, 1993), mediante la inducción del isoenzima 3A4 del sistema citocromo P450, responsable de su desmetilación e inhibir la isoenzima 2D6, responsable del sistema de biotransformación utilizado por numerosos fármacos ( Micó et al, 1999).

Por lo tanto hay muchos factores que pueden modificar la efectividad farmacológica de la metadona (Kreek,1973), entre los que destacan las hepatopatías, tan frecuentes en estos pacientes, enfermedades renales crónicas, así como interacciones a nivel farmacocinético o farmacodinámico debido al uso concomitante de otras drogas o medicamentos para el tratamiento de otras enfermedades.

En los pacientes adultos sin disfunción hepatorrenal, el aclaramiento de la metadona es de 1,4 $\mathrm{ml} / \mathrm{min} / \mathrm{kg}$. Aparece inalterada en orina en un $24 \%$ y el $76 \%$ restante es atribuible a sus metabolitos.

La cantidad de metadona inalterada excretada por la orina es $\mathrm{pH}$-dependiente: aumenta cuando ésta es acidificada, ya que es una base débil (Jaffe, 1990) y (Kreek, 1979). Diferentes estudios han mostrado que transformando un $\mathrm{pH}$ muy ácido en un $\mathrm{pH}$ muy alcalino, la vida media de la metadona puede aumentar de menos de 18 horas a más de 40 horas.

\section{LAAM}

EI LAAM es un agonista opioide sintético, que actúa preferentemente en los receptores "mu" con acciones similares a la morfina y la metadona. Actúa inicialmente en el sistema nervioso central y sistema gastrointestinal con efectos muy diversos, entre los que se incluyen analgesia, sedación, constricción pupilar, depresión respiratoria, disminución de la motilidad gastrointestinal y cambios en la función neuroendocri- na y en la función del sistema nervioso autónomo (Fraser e Isbell 1952),( Fraser, et al. 1954), ( Jaffe y Martin, 1985). Como con otros opiáceos, el uso repetido de la droga acaba provocando tolerancia (Ling y Rawson, 1997).

Las características farmacocinéticas del LAAM han sido ampliamente estudiadas (Henderson, 1976), (Kaiko,1975), (Quinn ,1977), (Moody, 1977), (Walsh, 1998), (San ,1997), Walsh et al 1998 describen la disponibilidad del LAAM y sus metabolitos en plasma después de la administración por vía oral e intravenosa en humanos. Tras la administración intravenosa, se observa una rápida desaparición del LAAM del plasma, sugiriendo que el fármaco se distribuye rápidamente a otros compartimentos donde puede permanecer debido a su gran liposolubilidad. Por el contrario, en solución oral, LAAM se absorbe rápidamente por el tracto gastrointestinal, detectándose en plasma a los 30 minutos, alcanzando su pico de concentración máxima a las 2-4 horas (steady-state) (Walsh et al, 1998) .

La biodisponibilidad, estimada por comparación de las áreas bajo la curva obtenidas tras la administración oral e intravenosa, es del $48 \%$ para LAAM, respecto al casi $100 \%$ definido para metadona. Esta baja biodisponibilidad se justifica por el efecto del primer paso hepático que sufre el LAAM tras la administración oral y los datos recientes de estudios metabólicos realizados in vitro, sugieren que es el citocromo P450 3A4 el enzima primariamente implicado en su metabolismo o degradación (Moody, 1997).

De hecho, debido a este efecto de primer paso y mediante un proceso de N-demetilación, LAAM se transforma sucesivamente en nor-LAAM y dinorLAAM, secuencia metabólica que se hace evidente por el orden de aparición de los metabolitos y su desaparición secuencial del plasma (Walsh, 1998). Aunque la N-demetilación es la principal ruta metabólica del LAAM, también se han descrito otras vías menores de eliminación que comprenden la excreción directa y la desacetilación a metadol, normetadol y dinormetadol (Billings, 1973).

Los hallazgos en relación a la enzima P450 3A4 como responsable de la N-desmetilación de LAAM y metadona, abren camino para comprender las múltiples interacciones medicamentosas producidas por inhibición o inducción que deben ser consideradas a lo largo del tratamiento con todos los fármacos que utilizan para su biotransformación este sistema enzimático (Moody et al, 1997).

En conjunto, el principio activo LAAM tiene una semivida plasmática de aproximadamente 62 horas (Iturrisi y Verebely 1972), (Sawe, 1986), (Inturrisi, 1987), a diferencia de las 30 horas de la metadona. Esto se debe a que la metadona se metaboliza principalmente en metabolitos inactivos (Sullivan, 1973), 
mientras que LAAM, como se ha mencionado anteriormente, se metaboliza a dos compuestos farmacológicamente activos, siendo nor-LAAM de 5 a 10 veces más potente que LAAM y dinor-LAAM.

En relación a la semivida del LAAM, se estima en 46 a 47 horas mientras que las de sus metabolitos son de aproximadamente 62,4 horas para norLAAM y 174,6 horas para dinor-LAAM.( Walsh et al, 1998).

Son las semividas prolongadas de estos metabolitos, las que explican la prolongada actividad clínica y la duración de sus efectos, permitiendo proponer un tratamiento con LAAM administrado tres veces por semana (la vida media de eliminación: para LAAM es de 2,6 días, para nor-LAAM de 2,0 días y para dinorLAAM de 4,0 días). Esto determina que a las 72 horas de su administración las concentraciones plasmáticas que se obtienen para los 3 productos sean suficientes para mantener su actividad terapéutica (Bammer, 1997).

El aclaramiento renal del LAAM y sus metabolitos es $\mathrm{pH}$ dependiente, observándose una mayor eliminación en sujetos con orina ácida (Kaiko \& Inturrisi, 1975). Sin embargo, sería de esperar que sujetos con una orina alcalina tiendan a excretar una menor cantidad a través de la orina y consecuentemente subirían las concentraciones en plasma, pero todavía no se han realizado estudios que comprueben este hecho.

La excreción urinaria del LAAM y sus dos metabolitos desalquilados expresada como el porcentaje del total de la dosis administrada, es menor de un 20 a $25 \%$, aunque existen diferencias interindividuales y un 1,6-1,7\% se excreta en forma de metadol, normetadol y dinormetadol.

Por su estructura química, LAAM y sus metabolitos, pueden conjugarse con ácido glucurónico y excretarse por vía biliar; como consecuencia y debido a la circulación enterohepática, se produce una prolongación de la actividad sistémica de este fármaco.

Tras la suspensión de metadona o LAAM, los síntomas de abstinencia a opiáceos comienzan a aparecer a las 72 horas en el caso de LAAM a diferencia de las 24 horas de la metadona (Ling et al,1978).

\section{INTERACCIONES DE LA METADONAY LAAM CON OTROS FÁRMACOS}

La utilización de metadona o LAAM junto con fármacos inductores o inhibidores metabólicos puede suponer que en un momento determinado, tras la administración del fármaco aumenten rápidamente o disminuyan los niveles plasmáticos de metadona o LAAM, produciéndose sobredosificación o síndrome de abstinencia.

\section{METADONA}

Hay muchos factores que pueden modificar en teoría la efectividad farmacológica de la metadona (Kreek, 1973). Algunos fármacos utilizados habitualmente en combinación con la metadona debido a enfermedades concomitantes, aceleran el metabolismo de la metadona por inducción de los sistemas microsomales hepáticos y provocan una bajada de los niveles de la misma(Wolff, 1991).

El comienzo de la acción inductora es gradual, y el final del efecto dependerá de la eliminación del fármaco inductor y la restauración del nivel normal del enzima.

Así ,fármacos como los anticonvulsivantes (barbitúricos, carbamacepina y fenitoína) (Tong et al, 1981), fármacos antituberculosos (rifampicina) (Kreek, 1976), nuevos antirretrovirales como la nevirapina y el efavirenz (Heelon y Meade, 1999), (Altice et al, 1999), ( Otero et al, 1999), (Baño et al, 2000), favorecen la biotransformación y por tanto la velocidad de eliminación de metadona.

Por este efecto, el paciente comienza con síndrome de abstinencia que obliga al aumento de dosis progresivo y como consecuencia se producen alteraciones fisiológicas, como pérdida de peso, diarrea (Goode, 1971) y alteraciones metabólicas ( en las proteínas plasmáticas) (Garrido et al, 1999), que pueden alterar la cinética de la metadona, cambiando la eficacia del fármaco por una modificación en el volumen de distribución y variación de su concentración en la biofase.

También hay que tener en cuenta que, según algunos autores, la disminución de las concentraciones de metadona puede ser, en algunos casos atribuida a un incremento del propio catabolismo, por la inducción enzimática debida a un aumento de la síntesis del enzima, en pacientes que están con el tratamiento mucho tiempo (Nilson et al, 1982), (Wolff et al, 1991) o la aparición de anticuerpos contra la metadona (Gamaleya et al, 1996) (Gamaleya et al, 1999).

Otros fármacos tienen un efecto inhibidor del metabolismo, produciendo un aumento de las concentraciones de metadona por bloqueo del citocromo P450. Este es el caso de las benzodiacepinas, antidepresivos y antipsicóticos, que potencian el efecto elevador de la metadona.

\section{LAAM}

En el caso del LAAM, el tratamiento concomitante con fármacos inductores no debería tener casi efecto clínico ya que al favorecer el paso de LAAM a nor LAAM y dinor LAAM y ser sus metabolitos más acti- 
vos, el resultado final debería ser suficiente para mantener al paciente estabilizado sin síntomas de abstinencia.

Con fármacos inhibidores del metabolismo, la inhibición de la N-desmetilación aumenta la potencia agonista de opioides que tienen normetabolitos inactivos como ocurre con la metadona, pero también disminuyen la potencia agonista de opioides que tienen normetabolitos más potentes (p.ej.: LAAM). En cualquier caso todos los pacientes en tratamiento con agonistas opiáceos que tomen fármacos que inhiban el metabolismo se les debe advertir del riesgo de sobredosis.

\section{MONITORIZACIÓN TERAPEUTICA (MT) DE META- DONA Y LAAM}

\section{INDICACIONES DE MT PARA METADONA}

Existen evidencias cada vez más crecientes de que las estrategias de monitorización de la concentración de fármacos en la evaluación del tratamiento pueden facilitar el descubrimiento de la dosis óptima y el mejor tipo de tratamiento para cada caso (Wolff et al,1997). Los niveles plasmáticos de metadona, más que una evaluación de la dosis- respuesta, representan una estrategia para obtener una información racional sobre las dosis que ayuden a los pacientes a estar bien con la metadona (Wolff, 1997), (Hiltunen et al, 1995) (Torrens et al,1998). Por tanto, los "niveles plasmáticos adecuados", más que las dosis adecuadas pueden utilizarse como instrumento clínico para valorar la eficacia del tratamiento con metadona y para detectar alteraciones en pacientes estabilizados (Baño et al, 2000).

Las diferencias interindividuales de dosis pueden deberse al grado de tolerancia a opiáceos de cada paciente, a variaciones farmacocinéticas, como por ejemplo, un excesivo aclaramiento de metadona, incluyendo en este grupo los metabolizadores rápidos e interacciones medicamentosas, con fármacos inductores enzimáticos.

Sin embargo, en el momento actual son pocos los programas de mantenimiento con metadona que utilizan la monitorización terapeútica en sus programas de tratamiento, aunque diversos estudios demuestran que los niveles plasmáticos de metadona son la mejor medida para la evaluación de los pacientes (Wolff, 1991, 1997),( Baño et al, 1998 y 2000).

\section{VENTAJAS E INCONVENIENTES DE LA MT EN METADONA}

Es evidente que la monitorización terapeútica tiene la ventaja potencial de permitir una mayor flexibilidad en las dosificaciones e individualizarlas, con la seguridad de dar dosis altas sin problemas (Wolff et al,1991), descubriendo sobre o infradosificaciones (Hubbard,1989) y detectando cumplimientos escasos (Wolff et al,1991). Por otra parte, permite valorar la influencia de factores exógenos ( hábitos nutricionales, estilo de vida), endógenos (enfermedades concomitantes, embarazo, sexo) así como posibles interacciones medicamentosas que pueden sufrir los pacientes y cuyas consecuencias pueden ser fundamentales tanto para la adhesión al programa, como para la salud del propio paciente.

Otra ventaja es poder monitorizar el cumplimiento del tratamiento, ya que muchos adictos a opiáceos no realizan una adecuada adherencia a la terapia (Wolff et al, 1991). Para poder valorar el grado de cumplimiento es fundamental saber si el paciente toma toda la medicación correctamente y descubrir si usan dosis extras o no toman parte de su prescripción.

En estudios realizados con metadona se ha observado que cuando el cumplimiento es bueno, existe una buena correlación entre dosis y concentraciones plasmáticas de metadona (Dole 1991, Loimer 1992, Baño et al, 2000) permitiendo utilizar los niveles de metadona para validar las dosificaciones.

En cuanto a los inconvenientes de su uso, la principal desventaja es que hay que realizar flebotomías a pacientes que tienen sus venas muy dañadas y se necesita un personal de enfermería entrenado, el aparataje es caro y requiere personal de laboratorio altamente especializado para su realización. Los costes según las técnicas empleadas pueden ser elevados. Utilizando técnicas de EIA (Guillén et al, 1998), los costes disminuyen y se pueden procesar mayor número de muestras. Por otra parte la interpretación de los resultados requiere conocimientos especializados o asesoramiento de clínicos entrenados ( Baño et al, 1998)

\section{VENTAJAS E INCONVENIENTES DEL USO DE LAAM}

Las ventajas del uso de LAAM están relacionadas con su lenta aparición en plasma tras su administración oral y su larga duración de acción, produciendo un efecto más estable con menos fluctuaciones. Algunos pacientes describen que se sienten "más normales", especialmente aquéllos que no toleran bien la metadona (Karp- Gelernter et al,1982) ( Savage et al, 1976),(Resnick et al, 1981). El tratamiento sólo será necesario tres veces a la semana en comparación con la toma diaria que necesitaban de metadona (Rawson et al, 1998). Su uso intravenoso entre la población adicta no es probable, debido a la rápida desaparición del LAAM del plasma por su alta distribución, disminuyendo la posibilidad de tráfico de esta sustancia. Un estudio publicado recientemente por Rolley et al, 
compara el uso de metadona, buprenorfina y LAAM en el tratamiento de la dependencia opioide, demostrando que el LAAM es el que produce una mayor abstinencia (Johnson et al, 2000).

Los diferentes estudios realizados para evaluar el efecto del LAAM en la función hepática, posible toxicidad, alteraciones hematológicas o urinarias, indican que los resultados obtenidos son similares a aquellos efectos que aparecen en los grupos de metadona o población consumidora de heroína (Jaffe et al, 1972), (Savage et al, 1976), (Ling et al, 1978), (Tennant et al, 1986). No existen evidencias hasta la fecha que sugieran hepatotoxicidad o afectación de la función renal por el uso de LAAM, aún así debería usarse con precaución en pacientes con enfermedades hepáticas o con alteraciónes en la conducción cardíaca por el riesgo de arritmias (Ling et al,1994) No se han encontrado efectos secundarios diferentes del uso de la metadona. En general los más habituales pueden ser estreñimiento, sudoración excesiva, astenia, nerviosismo y dificultades para dormir, y los estudios realizados para analizar las diferencias entre el uso de la metadona y LAAM demuestran que son escasas y que hasta la fecha el uso de LAAM es tan seguro como el de metadona para el manejo de la dependencia a heroína (Kutin y Lintzeris, 1997)

\section{MT DE LAAM}

Aunque los niveles en plasma de LAAM varían considerablemente, las concentraciones en plasma de sus metabolitos son mucho más estables, especialmente en el caso del dinorLAAM (Ling, 1994).Un estudio metabólico realizado en ratones y ratas (Sung y Way, 1954) describen que el proceso de conversión del LAAM en sus metabolitos activos ocurre más rápidamente después de la administración oral que tras la administración parenteral; además la primera N-demetilación ocurre aproximadamente 3 veces más rápido que la segunda, por lo que el metabolito norLAAM tiende a acumularse (McMahan et al, 1965). Este efecto acumulativo puede ser potenciado en un principio por la propia molécula de norLAAM, debido al efecto bifásico que ejerce en el sistema microsomal hepático, actuando primero como inhibidor mediante inactivación del enzima por formación del complejo citocromo P-450-norLAAM, produciéndose la síntesis compensatoria de novo (Roberts y Franklin , 1979).

Este mecanismo bioquímico puede jugar un papel importante en las interacciones medicamentosas en pacientes tratados con LAAM.

En algunos individuos, la evolución de las concentraciones plasmáticas en el tiempo durante la administración continuada del LAAM aumenta durante todo el período de dosificación, produciéndose en algunos casos la acumulación de las tres sustancias, en otros se acumula LAAM y dinor LAAM y sólo en alguno dinorLAAM. Esta acumulación no presenta una relación directa con la dosis. El factor de incremento para las concentraciones plasmáticas del LAAM, después de la última dosis respecto a las concentraciones alcanzadas tras la primera dosis, fue de 1,2-2 para LAAM, 1,4-2 para nor-LAAM y 3-4 en el caso del dinorLAAM; estos incrementos son mayores en los individuos acumuladores. El efecto acumulativo puede evitarse si administramos LAAM en dosis menores de $0,86 \mathrm{mg} / \mathrm{kg}$ y a intervalos mayores de 48 horas (Henderson et al, 1976) a dosis de $1 \mathrm{mg} / \mathrm{kg}$ tres veces por semana (Finkle et al, 1982).

Debido a la gran liposolubilidad y alto volumen de distribución, la concentración estable se alcanza aproximadamente entre la tercera y quinta dosis en el caso de pacientes que siguen una cinética normal sin acumulación de metabolitos, es decir, después de 3 ó 4 dosis ó 218 horas para LAAM; 3 a 4 dosis ó 196 horas para norLAAM y de 3 a 6 dosis ó 300 horas en el caso del dinorLAAM.

La realización de niveles en plasma de LAAM podría ser de gran utilidad en el manejo del paciente al igual que ocurre con los de metadona. Sin embargo, hay que tener en cuenta que cuando se alcanza el estado estacionario la concentración de metabolitos es más estable sobre todo en el caso de dinorLAAM (Ling et al, 1994). Este momento se puede determinar mediante los niveles plasmáticos, siguiendo los datos recogidos en la literatura: de 3 a 4 semividas para el LAAM y 10 semividas para metadona. La mayoría de los pacientes tratados con LAAM se estabilizan en el intervalo de dosis comprendido entre 50 y $90 \mathrm{mg}$ tres veces por semana, siendo el intervalo entre las dosis habitual de 48 horas y uno de ellos de 72 horas. Esto obliga a un ajuste diferente de dosificación que no siempre es sencillo.La determinación de los niveles plasmáticos residuales, en este tipo de pacientes, después de 48 o 72 horas antes de la toma siguiente, nos podría dar información acerca del ajuste de dosis más adecuado.

Hasta la fecha, no existen estudios de aplicación clínica de niveles en plasma de LAAM y sus metabolitos, sólo estudios experimentales.

\section{POSIBLES INDICACIONES TERAPEÚTICAS DE LAAM}

A menudo, para referirnos al LAAM se utiliza el término "metadona de larga duración". Esta comparación con metadona es errónea, ya que LAAM por sí mismo ofrece un débil efecto opioide y en su conjunto presenta unas características farmacológicas propias, por lo que su uso requiere el desarrollo de una estrategia de tratamiento. 
Los estudios publicados hasta el momento actual hablan de buenos y malos candidatos para el uso de LAAM, entendiendo por "buenos candidatos" pacientes estabilizados en metadona, con empleo y una actitud positiva hacia el tratamiento y "pobres candidatos", todos aquellos que toman medicación concomitante padecen alguna enfermedad hepática o renal o consumen otras sustancias como alcohol o benzodiacepinas (Rawson y cols, 1998). Es decir, bajo este punto de vista, muy pocos de los pacientes habituales de los programas de mantenimiento con metadona en España podrían beneficiarse del uso del LAAM.

Como alternativas terapeúticas a estos criterios, basados en la farmacología de este agonista y en los estudios realizados por este equipo sobre niveles en plasma de metadona, se proponen otros perfiles de uso de LAAM y su monitorización terapeútica:

1.- Pacientes que solicitan programas de metadona (PMM) de corta duración. Perfil social adaptado, buen pronóstico, sin patología orgánica grave y que desean un programa de agonistas orientado al cambio con desintoxicación y posterior paso a programa libre de drogas. La inducción y estabilización inicial se realizaría con metadona y posteriormente, previa realización de los niveles en plasma, se sustituiría por LAAM . El objetivo sería psicosocial, orientado a normalizar su estilo de vida , aprovechando el efecto clínico más importante del LAAM a nivel cerebral ( " se sienten más normales o más confortables"). Es decir, el tener menos picos, subidas o "high" como ocurre con otros agonistas, les permitiría el uso del mismo sin sentir cambios en el sistema nervioso central, facilitando la normalización de su vida.

2.-Pacientes en PMM estabilizados, con buena respuesta al tratamiento y a los que se desearía flexibilizar y espaciar la administración diaria de metadona sin tener que recurrir a la problemática práctica de medicación para llevar a casa (take home). Se realizarían niveles de metadona previos para ajustar correctamente el paso de metadona a LAAM. Este es el perfil que se recomienda en los trabajos americanos realizados por Rawson et al, (1998) y en pacientes considerados como "buenos candidatos". Nuestros pacientes, cuando están estabilizados en metadona, recogen dosis 2 veces a la semana y el cambio a LAAM les supone una desventaja añadida al tener que recoger el fármaco 3 veces por semana.

3.- Pacientes en PMM que pierden la estabilidad.

Después de haber conseguido la estabilización durante un tiempo que puede ser variable en cada caso, algunos pacientes comienzan a quejarse de síndrome de abstinencia a opiáceos que no remite a pesar de incrementar sus dosificaciones. Además de los síntomas clínicos se observa que sus niveles en plasma disminuyen sin encontrar ninguna causa que lo justifique, exluyendo la autoinducción, la toma incorrecta o cambios en la medicación concomitante que toman habitualmente. En estos casos, el paso a LAAM se hará según el nivel de metadona previo al cambio y la dosis que le correspondería para ese nivel según rangos establecidos de dosis/niveles por ( Baño et al, 2000).

4.- Pacientes en PMM que toman fármacos que pueden producir "Interacciones medicamentosas".

Basados en la información farmacocinética existente sobre LAAM, sería posible predecir las interacciones para una gran cantidad de medicamentos asociados al uso del LAAM y sus posibles efectos. En el caso de fármacos inductores del metabolismo de la metadona anteriormente mencionados, y metabolizadores rápidos, favorecerían la biotransformación y por tanto la velocidad de eliminación de esta sustancia.

En el caso del LAAM y debido a que sus metabolitos son más activos, la caída del nivel quedaría compensada con la mayor potencia del norLAAM y dinorLAAM, aunque puede disminuir la duración del efecto habitual y ser necesarios aumentos de dosis. De esta forma, un problema como es la caída del nivel de metadona (metabolitos inactivos) tras el tratamiento con inductores se puede transformar en una oportunidad para estabilizar a los pacientes con LAAM (metabolitos activos y de mayor semivida) (Baño et al, 2000).

En estos casos debería contemplarse como indicación terapeútica el paso a LAAM temporalmente mientras el paciente permanezca con el medicamento inductor, con el objetivo de mantener más tiempo los niveles plasmáticos y controlar el síndrome de abstinencia a opiáceos (SAO).

Es preciso realizar un nivel previo de metadona que verifique que el paciente presenta unos niveles en plasma muy bajos ( $<100 \mathrm{ng} / \mathrm{ml}$ ) a pesar de dosis altas (> $100 \mathrm{mg}$ ). El paso a LAAM se hará en función del nivel en plasma previo de metadona y la dosis de metadona que le correspondería para ese nivel. A partir de aquí se irá aumentando la dosis de LAAM hasta conseguir la estabilización del paciente sin síntomas de abstinencia ni sobredosificación.

\section{CONCLUSIONES}

El manejo de los agonistas opiáceos en los programas de mantenimiento y la búsqueda de indicaciones 
clínicas adecuadas para cada paciente debe ser uno de los objetivos de todos los clínicos que trabajan en estos programas para conseguir su estabilización y una mejoría en su calidad de vida.

Las diferencias farmacológicas entre ambos agonistas son las que definen perfiles de uso diferentes. EI LAAM, debido a su lenta aparición en plasma y su larga duración de acción produce un efecto más estable, con menos fluctuciones. En base a este efecto se ha utilizado en pacientes estabilizados con la metadona para espaciar su administración como única indicación clínica. En este artículo se proponen otras posibilidades, pudiendo estar indicado en programas de alto umbral, orientados al cambio en un corto período de tiempo, con desintoxicación y paso posterior a programas libres de drogas aprovechando el efecto de estabilidad que hace que los pacientes se sientan "más normales" que con la metadona. Otro grupo de indicaciones de uso estaría en relación con los pacientes que pierden estabilidad en los programas de metadona, demostrado a través de la aparición de síntomas de abstinencia a opiáceos y disminución de los niveles en plasma, producido por interacciones medicamentosas $u$ otros motivos no siempre conocidos. El paso a LAAM, en estos casos, al ser sus metabolitos más activos que los de metadona, compensaría la caída del nivel, aunque puede disminuir la duración del efecto habitual y ser necesarios ajustes de dosis.

En cualquier caso, el uso de agonistas opiáceos en programas de mantenimiento debe individualizarse en cada paciente, teniendo en cuenta que la metadona se utiliza como tratamiento de inicio en todos ellos y el LAAM representa una alternativa terapeútica en algunos casos. Serán necesarios estudios con los perfiles propuestos que amplíen el uso del LAAM para pacientes que tienen problemas con la metadona, intentando utilizar la monitorización en plasma como un instrumento de ayuda y evaluación que mejore la eficacia de los mismos.

\section{BIBLIOGRAFÍA}

- Altice F.I., Friedland G.H., Cooncy E.L.(1999) Nevirapine induced opiate withdrawal among injection drug users with HIV infection receiving methadone. AIDS Vol. 13: 957-962.

- Ball J.C, Lange R.W, MyersC.P.(1988)Reducing the risk of AIDS through methadone maintenance treatment. Journal Of Health and social behavior. 29:214-226.

- Baño M.D.,Agujetas M., López M.L., Millán I., Lora Tamayo C., Guillén J.L.(1998). Resultados del estudio piloto realizado en el Programa Municipal de Drogas de Majadahonda sobre niveles plasmáticos de metadona. En: Cabrera J.,Guillén J.L.eds. La huella de la metadona:Niveles plasmáticos. Un instrumento clínico para mejorar tratamientos. Madrid Delos S.L.123146.

- Baño M.D., Agujetas M., López M.L., Guillén J.L.(2000) La nevirapina induce síntomas de abstinencia en pacientes en programa de mantenimiento con metadona con infección VIH. Revista Clinica Española. 200 (1):18-20.

- Baño M.D., López M.L, Agujetas M., Millán I., Guillén J.L.(2000). Niveles plasmáticos de metadona. Resultados después de 15 meses de seguimiento en el Programa Municipal de Drogas de Majadahonda. Adicciones."en prensa".

- Bammer G. (1997) Practical issues of pharmacotherapy availability and trialing Chapter III. In: Ritter A., Kutin J., Lintzeris N., Bammer G.(eds). Expanding treatment options for heroin dependence in Victoria: buprenorphine, LAAM, naltrexone and slow release oral morphine. New Pharmacotherapies ProjectFeasibility Phase. Fitzroy, Victoria: Turning point alcohol and drug centre Inc.

- Billings RE.(1973). Metabolism of acetlymethadol. A sensitive assay for nor-acetylmethadol and the identification of a new active metabolite. J.Med Chem . 16: 305-306.

- Brugal MT,Puigdollers E (1999).Evluación de la efectividad de diferentes subprogramas de metadona. Adicciones. 11(1):6-7.

- Finkle, B.S., Jennison, T.A., Chinn, D.M., Ling, W., Holmes, E.D. (1982).Plasma and urine disposition of 1-alphaacetylmethadol and its principal metabolites in man. Journal of Analytical Toxicology. 6: 100-105.

- De Castro J. (1996). The effects of changes in gastric $\mathrm{pH}$ induced by omeprazole on the absorption and respiratory depression of metadone.Biopharm-DrugDispos. 17(7): 551-63

- Dole V.P. Foreword (1991). In The efectiveness of methadone maintenance treatment. Ball J.C and Ross A. Eds. Springer, New york. VII-VIII.

- Fraser H.F, Isbell H.F. (1952) Actions and addiction liabilities of alpha-acetylmethadol in man. J. Pharmacol Exp ther. 105: 458-465.

- Fraser, H.F., Nash,T.L., Vanhorn, G.D., Isbell,H.(1954) Use of miotic effect in evaluating analgesic drugs in man. Archives Internationales des Pharmacodynamie et de Therapie. 98:443-451

- Freedman R.R, \& Czertko, G.(1981).A comparison of thwice weekly LAAM and daily methadone in employed heroin addicts. Drug and Alcohol Dependence.8(3): 215-222.

- Gamaleya N, Tronnikov S, Ulyanova L, Klimova S; Dmitrieva I.(1996) Antibodies to morphine and impaired immune reactivity. Addict Biol. 1: 437-445

- Gamaleya N, Dmitrieva I, Borg S, Ericson N.(1999) Induction of antibodies to methadone during methadone maintenance treatment of heroin addicts and its possible clinical implications. Eur. J. Pharmacol. 26:369 (3):357-64

- Garrido MJ, Valle M, Calvo R, Troconiz IF.(1999).Altered plasma and brain disposition and pharmacodynamics of 
methadone in abstinent rats. J Pharmacol Exp Ther. Jan. 288 (1):179-87.n

- Glass R.M(1993): Methadone maintenance: New research on a controversial treatment(editorial). JAMA. 269:1995-1996.

- Goode PG (1971).And implanted reservoir of morphine solution for rapid induction of physical dependence in rats. Br J Pharmacol. 41:558-566.

- Guillén JL., López ML(1998)Técnica analítica para la determinación de niveles plasmáticos de metadona. En: CabreraJ.Guillén JL.eds. La Huella de la Metadona. Niveles plasmáticos. Un instrumento clínico para mejorar tratamientos. Delos. S.L. Madrid .457-472.

- Hall W, Bell J, Carless J.(1993) Crime and drug use among applicants for methadone maintenance. Drug Alcohol Dependence.31:123-129.

- Heelon M.W., Meade LB.(1999)Methadone withdrawal when starting an antiretroviral regimen including nevirapine. Pharmacotherapy. 19(4).471-2.

- Henderson GL, Wilson BK, Derick HM. (1976). Plasma Ialpha-acetylmethadol after acute and chronic administration. Clin.Pharmacol.Ther. 21: 16-25.

- Hiltunen AJ.,Lafolie P, Martel J, Ottosson F-C Boreus L.O, Beck O, Borg S, Hjemdahl P. (1995) Subjetive and objective symptoms in relation to plasma methadone concentration in methadone patients. Psychopharmacology. 118: 122-126.

- Hubbard RL, Marsden ME., Rachal JV, Harwood HJ, Cavanaugh ER, Ginzburg HM.(1989) Drug Abuse Treatment: A National Study of Effectiveness. Chapell Hill, Universty of North Carolina Press.

- Inturrisi CE, Verebely K.(1972). The levels of methadone in the plasma in methadone maintenance. Clin. Pharmacol Ther. 13: 633-637.

- Inturrisi C.E. (1987).Pharmacokinetics and pharmacodynamics of methadone in patients with chronic pain. Clin Pharmacol. Ther. 41: 392-401.

- Jaffe, J.H., Senay, E.C. Schuster, C.R.(1972) .Methadyl acetate vs. methadone: a double-blind study in heroin users. Journal of the American Medical Association 3: 21-26.

- Jaffe JH, Schuster CR, Smith BB, Blachyl PH.(1976). Comparison of acetyl methadol and methadone in the treatment of long term heroin users: A pilot study National Institute on Drug Abuse Research Monograph. $N^{\circ}$ 8, Washington, DC, Government Printing Office, 72-73.

- Jaffe JH, y Martin W.(1985) Opioid analgesics and antagonists. In A.G.Gilman L.S.Goodman,T.W. Rall y F.Murad (Eds). Goodman and Gilman's .The pharmacological basis of therapeutics 7Th.ed. N.Y:Macmillan. 491-531.

- Jaffe J.H.(1990) Drogadicción y abuso de drogas En : Bases farmacológicas de la terapeútica. Goodman y Gilman eds. Madrid Ed. Panamericana. 513-560.

- Johnson R, Chutuape MA, Strain E, Walsh S, Stitzer M, Bigelow E. (2000) A comparison of levomethadyl acetate, buprnorphine, adn methadone for opioid dependence. The New England Jorunal of Medicine. 343(18) : 1290-1297

- Kaiko RF, Inturrisi CE. (1975) Disposition of acetylmethadol in relation to pharmacologic action. Clin. Pharmacol Ther. 18: 96-103.

- Kang SY, De Leon G. (1993) Correlates of drug injection behaviours among methadone outpatients. Am.Drug Alcohol Abuse. 19:107

- Karp-Gelernter, E., Savage C., McCabe, O.L.(1982). Evaluation of clinic attendance schedules for LAAM and methadone a controlled study. International Journal of the Addictions. 17: 805-813.

- Kreek MJ.(1973) Plasma and urine levels of methadone. NY State J. Med.73: 2773-2777.

- Kreek MJ.(1976)Rifampicin- induced methadone withdrawal. New England Journal of Medicine. 294:11041106.

- Kreek MJ.(1979) Methadone in treatment physiological and pharmacological issues In: Handbook on drug abuse. Dupont R. Goldstein A. Eds.. Washington D.C. U.S. Govt. Printing Office.

- Kutin J, Lintzeris N.(1997). Introduction and background to the feasibility research. In: Expanding treatment options from heroin dependence in Victoria: buprenorphine, LAAM, naltrexone and slow release oral morphine. New pharmacotherapies projectfeasibility phase. Ritter A., Kutin J., Lintzeris n., Bammer $\mathrm{G}$ eds. Fitzroy, Victoria.Turning Point Alcohol and drug centre Inc. Chapter 1:1-10.

- Ling W. (1978) Levo-alpha-acetylmethadol (LAAM) for opiate addiction. Current Psychiatric Therapies. 18: 173180.

- Ling W (1994). Substitution pharmacotherapies for opioid addiction: from methadone to LAAM and buprenorphine. J Psychoactive Drugs. 26:119-128.

- Ling W y Rawson R.A. (1997).American opiate substitution treatment programs:From methadone to LAAM and Buprenorphine. In:.The medical prescription of narcotics: Scientific foundation and practical experiencies. D.Lewis C. Gear,M.Laubli Loud, y D. Langenic-Cartwright eds. Gottingen: Hogrefe y Huber. 250-263.

- Loimer N, Schmid R. (1992). The use of plasma levels to optimize methadone maintenance treatment. Drug and Alcohol Dependence.30.:241-246.

- López ML, Baño MD, Agujetas M, Guillén JL (2000). Algoritmo del seguimiento de niveles plasmáticos de metadona en pacientes HIV en tratamiento con nevirapina. Adicciones.12(1):109.

- McEvoy GK.(1990) AHFS Drug Information 90. American Hospital Formulary Serviced. Bethesda: American Society of Hospital Pharmacists Inc.

- MacMahan, R.F., Culp, H.W., \& Marshall, F.J.(1965) The metabolism of I-acetylmethadol in the rat: The identification of the probable active metabolite. Journal of Pharmacology and Experimental Therapeutics. 149: 436-445. 
- McLachlan C, Crofts N,Wodak A, Crowe S.(1993). The effects of mehtadone on immune function among injecting drug users: A review. Addiction .88:257-263.

- Micó Segura JA, Rojas Corrales MO, Moreno Brea M.R.(1999)Interacciones medicamentosas de la metadona. Farmaceúticos.50-54.

- Moody DE, Alburges ME, Parker RJ, Collins JM, Strong JM. (1997). The involvement of cytochrome P450 3A4 in the $\mathrm{N}$-demethylation of L-alfa-acetylmethadol (LAAM). NLAAM and methadone. Drug Met Disp . 25 (12): 1347-1353.

- Nightingale,S.L.(1993). Levomethadyl approved for treatment of opiate dependence. J.Am Med Assoc 270: 1290.

- Nilson MI, Meresaar U, Anggard E. (1982). Clinical pharmacokinetics of methadone. Acta Anesthesiol Scand .74, suppl: 66-69.

- Otero MJ. Fuertes A, Sanchez R., Luna G.( 1999). Nevirapine-induced withdrawal symptoms in HIV patients on methadone maintenance programme: An alert. AIDS Vol. 13.1004-1005

- Quinn DI, Wodak A, O"Day R, Colling JM, Strong JM. (1997).Pharmacokinetic and Pharmacodynamic principles of illicit drug and treatment of illict drug users. Clin Pharmacokinetic .33 (5): 344-400.

- Rawson, R.A., Ling, W. (1993).Levo-alpha-acetylmethadol (LAAM) for the Treatment of Opiate Dependence. A White Paper. Prepared for the California Office of Alcohol and Drug Programs.

- Rawson R.A., Hasson A.L., Huber A.M. Mccann M.J., Ling W. (1998) A 3- year progress report on the implementation of LAAM in the United States. Addiction . 93 (4): 533-540.

- Roberts M, Franklin M.R.(1979).Modification of hepatic microsomal oxidative drug metabolism in rats by the opiate maintenance drug acetylmethadol, proposxyphene and methadone. Life Science Washington D.C. 25:845-852

- Reno RR,Aiken LS.(1993).Live activities and life quality of heroin addicts in and out of treatment. Int J.Addict. 28:211-232.

- Resnick RB, Washton AM, Garwood J, Perzel J.(1981) LAAM instead of take-home methadone. In L.S. Harris (Ed.). Problems of drug dependence. NIDA Research Monograph, Rockwille, MD. National Institute on Drug Abuse. 41: 473-475.

- San L. (1997). El LAAM. Nueva alternativa en tratamientos de mantenimiento con sustitutivos". En: Tratamientos sustitutivos en dependencias a opiáceos. Metadona, LAAM, Heroína. Avances en farmacología de drogodependencias. Universidad de Deusto.37-56.

- Savage C, Karp EG, Curran S.F, Hanlon TE., McCabe O.L. (1976). Methadone/LAAM maintenance: a comparison study. Comprehensive Psychiatry. 17: 415-424.
- Sawe J. (1986). High-dose morphine and methadone in cancer patients: Clinical Pharmacokinetic considerations of oral treatmen. Clin Pharmacokinetic.11: 87106.

- Seivewright NA, Greenwood J.(1996). What is important in drug misuse treatment?.Lancet 373-375

- Shuster CR. (1991) The National Institute on Drug Abuese and methadone maintenance treatment. J.Psychoact. Drugs. 23:111-112.

- Stimmel B (1993). Intravenous drug use, methadone and AIDS. Ask not for whom the bell tolls. J.Addict Dis. 12:1.

- Sullivan HR, Due SI. (1973). Urinary metabolites of methadone, in maintenance subjects. J. Med. Chem. 16: 909-913.

- Sung C. \& Way FL. (1954)The fate of optical isomers of aacetylmethadol. Journal of Pharmacology and Experimental Therapeutics . 110: 260-270.

- Tennant FS, Rawson RA, Pumphrey E, Seecof R. (1986). Clinical experiences with 959 opioid-dependent patients treated with levo-alpha-acetylmethadol. J Subst Abuse Treat. 3: 195-202.

- Tong TG, Pond S, Kreek MJ, Jaffery N, Benowitz N(1981). Phenytoin induced methadone withdrawal. Ann. Intern. Med. 94: 349-351.

- Torrens M, Castillo C, San L, Moral E, González ML, De la Torre R (1998). Plasma methadone concentrations as an indicator of opioid withdrawal symptoms and heroin use in a methadone maintenance program. Drug and Alcohol dependence . 52:193-200.

- Walsh S.L, Johnson R.E, Cone E.J Bigelow G.E(1998). Intravenous and oral l-alfa-acetymethadol: Pharmacodynamics and Pharmacokinetic in humans. J. Pharmacol. Exp. Ther. 285: 71-82.

- Ward J, Mattick R, Hall W. ( 1992) Key issues in methadone maintenance treatment. Australia, University of Nes South Wales Press.

- Wolff K, Sanderson M, Hay A, Raistrick D. (1991). Methadone concentrations in plasma and their relationship to drug dosage. Clin Chem . 37: 205-209.

- Wolff K. Hay A. Raistrick D. Calvert R.(1993). Steady-state pharmacokinetics of methadone in opioid addicts. Eur. J. Clin. Pharm. 44:189-194.

- Wolff K, Rostami, Hodjegan A, Shires S, Hay A, Feely M, Calvert R, Raistrick D. (1997). The pharmacokinetics of Methadone in healthy subjects and opiate users. Clin Pharmacol . 44: 325-334.

- Wolff K, Strang J. (1999) Therapeutic drug monitoring for methadone: Scanning the horizon. Eur. Addict Res. 5: 36-42.

- Zannini A, Nizzoli U,Bussolati N.(1997).Valoración y seguimiento de los tratamientos con metadona:índice de retención,marcadores biológicos y comportamentales. Adicciones.9 (3):457-466. 